\title{
Value of Magnetic Resonance Imaging Features in Diagnosis and Treatment of Breast Cancer under Intelligent Algorithms
}

\author{
Shuang Liu $\mathbb{D}^{1},{ }^{1}$ Min Tang $\left(\mathbb{D},{ }^{2}\right.$ Shuqin Ruan $\mathbb{D}^{2},{ }^{2}$ Feng Wei $\mathbb{D}^{2},{ }^{2}$ and Jiaxi Lu ${ }^{2}$ \\ ${ }^{1}$ Department of Ultrasound, Chongqing Health Center for Women and Children, No. 120, Longshan Road, Yubei, \\ Chongqing 401147, China \\ ${ }^{2}$ Department of Oncology and Hematology, Chongqing General Hospital, University of Chinese Academy of Sciences, \\ No. 312, Zhongshan First Road, Yuzhong, Chongqing 400013, China \\ Correspondence should be addressed to Min Tang; quewenjuan@hospital.cqmu.edu.cn
}

Received 30 August 2021; Accepted 19 October 2021; Published 3 November 2021

Academic Editor: Gustavo Ramirez

Copyright $\odot 2021$ Shuang Liu et al. This is an open access article distributed under the Creative Commons Attribution License, which permits unrestricted use, distribution, and reproduction in any medium, provided the original work is properly cited.

\begin{abstract}
This study was to analyze the clinical application value of magnetic resonance imaging (MRI) image features based on intelligent algorithms in the diagnosis and treatment of breast cancer and to provide an effective reference assessment for breast cancer diagnosis. The MRI diagnosis model (ACO-MRI) based on the ant colony algorithm (ACO) was proposed, which was compared with the diagnosis methods based on support vector machine (SVM) and proximity (KNN) algorithm, and the proposed algorithm was applied to MRI images to diagnose breast cancer. The results showed that the accuracy, sensitivity, and specificity of the ACO-MRI model were greater than those of the KNN and SVM algorithm. Moreover, the specificity was statistically considerable compared with the two algorithms of KNN and SVM $(P<0.05)$. By comparing $1 / 5$ number of ants and the average gray path of the ACO-MRI model under $1 / 8$ number of ants, it was found that the average gray path value of $1 / 8$ number of ants was greatly higher than the average gray path value of $1 / 5$ number of ants $(P<0.05)$. The differences in the overall distribution of breast MRI imaging features among Luminal A, Luminal $\mathrm{B}$, HER-2 overexpression, and TN were compared. There were considerable differences in the overall distribution of the three breast MRI imaging features of the boundaries, morphology, and enhancement methods among the four groups $(P<0.05)$. In short, MRI image based on the intelligent algorithm ACO-MRI diagnosis model can effectively improve the diagnosis effect of breast cancer. Its image feature boundaries, morphology, and enhancement methods had good imaging features in the diagnosis of breast cancer.
\end{abstract}

\section{Introduction}

As the most common malignant tumor in Chinese women, the incidence of breast cancer is increasing year by year. With the younger age of onset, it has once become the main cause of death for many female cancer patients [1]. However, the medical community does not have the same general idea about the pathogenesis of breast cancer due to the uncertainty of its pathogenic factors and the unclear dominance of early clinical features. Therefore, most of the diagnosed patients have reached the middle and advanced stage, thus missing the best treatment opportunity for the disease $[2,3]$. So far, breast cancer has had a considerable impact on women's physical and mental health, and the World Health Organization has paid great attention to it and paid close attention to its development trend [4]. How to improve the public awareness of breast cancer prevention and how to improve the early diagnosis and treatment of breast cancer have become an urgent work in China's breast cancer prevention and treatment [5].

Clinical diagnosis of breast cancer mainly involves imaging examinations, including ultrasound imaging, mammography (MAM), and breast magnetic resonance imaging (MRI) [6]. The principle of ultrasonic imaging diagnosis mainly uses the characteristics of ultrasonic wave, such as rapid-fire, reflection and refraction, and ultrasonic attenuation. This imaging is effective for the detection of substantive and cystic masses in the breast and can reflect the situation of axillary lymph nodes and surrounding tissues. Moreover, with the popularity of Doppler ultrasound 
imaging technology, its imaging performance is greatly improved [7]. However, due to the defect of unclear images in ultrasound, the detection probability of small breast lesions such as intraductal carcinoma is low [8]. The principle of breast MRI is magnetic resonance. The hydrogen protons detected in the human body emit electromagnetic waves under the action of external magnetic field so as to obtain the internal structure of the imaging object [9]. Compared with ultrasound, MRI has higher resolution and finer image, which can also image various parts of the human body from multiple faults and angles. From the early plain scanning to dynamic enhanced MRI scanning, the sensitivity and specificity of this detection method in the diagnosis of breast cancer have gradually increased. However, it still has limitations in the diagnosis of breast cancer. To improve the accuracy of its diagnosis, domestic and foreign experts have conducted massive studies [10].

The intelligent algorithm is a calculation method proposed by humans in the continuous pursuit and exploration of solutions, also known as soft computing. This method includes many contents, such as artificial neural network technology (ANN), genetic algorithm (GA), simulated annealing technology, and swarm intelligence technology. The swarm intelligence technology has derived the ant colony algorithm (ACO) and particle swarm optimization (PSO) $[11,12]$. Among the many algorithms, ACO and genetic algorithm can directly manipulate structural objects and can perform calculations and global optimization. This feature has a good development in imaging. With the popularization of artificial intelligence in the medical field, ACA also has a cross-combination in the field of medical imaging [13].

In summary, intelligent algorithms are more and more widely used in the field of medical imaging, but there are fewer applications in MRI diagnosis and treatment of breast cancer $[14,15]$. Therefore, MRI image feature diagnosis and treatment of breast cancer based on the ACO algorithm were proposed in this research, which was compared with the KNN algorithm and the SVM algorithm and was applied to the breast MRI examination of patients. By comparing the diagnostic performance of different MRI imaging feature parameters for breast cancer, the clinical application value of MRI imaging features based on intelligent algorithms in the diagnosis and treatment of breast cancer was comprehensively evaluated. It was expected that the combination of intelligent algorithm technology and magnetic resonance imaging technology can provide an effective reference for the clinical diagnosis and treatment of breast cancer.

\section{Materials and Methods}

2.1. Research Objects. In this study, 175 patients with breast problems who came to hospital for examination from July 2018 to July 2020 were selected as the research objects. Among them, 20 were male and 155 were female. All patients underwent MRI examination. This study had been approved by the Ethics Committee of hospital. Patients and their families had been aware of this study and had signed informed consent.
Inclusion criteria were as follows: (i) patients with complete clinical baseline data; (ii) patients who signed informed consent; (iii) patients who were confirmed by histological examination; and (iv) patients had not received any medical treatment. Exclusion criteria were as follows: (i) patients with major diseases and pregnant women; (ii) patients with hypersensitivity to contrast enhancement agents; and (iii) patients combined with tumors of other sites.

2.2. MRI Examination and Imaging Features. All patients underwent MRI examination. The breasts on both sides were placed in the inspection cavity in the prone position, and regular MRI scans were performed. After a plain scan, the contrast agent Magnevist (dose $0.1 \mathrm{mmol} / \mathrm{kg}$ ) and $20 \mathrm{~mL}$ normal saline were injected intravenously. The scan was performed before injection and at 0 s, 67 s, 134 s, $201 \mathrm{~s}, 268 \mathrm{~s}$, and $335 \mathrm{~s}$ after injection. The total time was $8 \mathrm{~min} 1 \mathrm{~s}$.

MRI image analysis was performed by two radiologists for independent imaging diagnosis. If the results were inconsistent, the two people should discuss together to reach a consistent result. The DWI image was input into the MRI workstation to obtain the apparent diffusion coefficient (ADC) map and measure the ADC value. MRI image was input into the imaging workstation to measure the IER value of the lesion. IER = (peak signal intensity within three minutes after injection of contrast agent-signal intensity before injection of contrast agent)/signal intensity before injection of contrast agent $\times 100 \%$.

The tumor was segmented by manual segmentation to obtain imaging characteristics. Independent imaging diagnosis was made by two radiologists. The region of interest (ROI) was delineated on the tumor region of ADC map at the maximum level of tumor using ImageJ to obtain relevant image features. Related image characteristics were (i) morphology such as maximum transverse diameter, area, and area ratio; (ii) first-order gray characteristics such as energy, skewness, average value, range, and standard difference; (iii) texture features such as the characteristics of the gray level concurrent matrix; and (iv) filtering characteristics. After the patients completed the relevant examinations, tumor resection was performed according to the indications, and pathological examination was conducted to clarify the pathological characteristics of the patients. For the selection of ROI in the lesion area, vascular, calcification, fat, cavity, bleeding area, cystic degeneration, necrosis, and normal glandular tissue group were avoided.

Observation and analysis of MRI images included morphological manifestations and enhancement types of lesions. For morphological manifestations, the lesions were classified into round, irregular, and lobulated shapes. The boundary between lesions and surrounding tissues was clear or unclear. The edges were smooth, uneven, or burr. After enhancement, the edges of lesions were classified into uniform enhancement and nonuniform enhancement. The shape of breast malignant lesions was irregular or lobulated, and the boundary between lesions and surrounding tissues was not clear, with incomplete edges or short burrs. 
Enhancement scan showed annular, spotty, and strip uneven enhancement, which was called malignant sign. Benign pathological changes of mammary gland were kind of circle, with the boundary of surrounding organization clear, the edge was smooth, and enhance scan was uniform aggrandizement, called benign sign.

2.3. Mathematical Model of ACO. The clustering of ACO in intelligent algorithms is quite similar to the process of image recognition, so it is widely used in images. However, to enhance the diagnostic performance of breast cancer, a better way to process MRI images is needed. The principle of the ACO algorithm is inspired by the study of ant colony behavior in nature. The algorithm has the three characteristics of distributed, natural organization, and positive feedback. The basic idea is constructing artificial ants, to simulate the real ant behavior in nature and solve optimization problems in many fields.

The number of artificial ants in the ant colony is set to $m$, $d_{y e}$ represents the distance between the city $y$ and city $e$, and $\tau_{y e}(t)$ represents the residual pheromone concentration between $y$ and $e$ at time $t$. It is stipulated that the behavior of ant $k$ conforms to the following law. In path selection, the next location is selected with corresponding function probability according to the concentration of path pheromone. It cannot choose the path that has been taken as the next step, and a data table $\left(\operatorname{tabu}_{F}(F=1,2,3,4, \ldots, m)\right.$ is used to control it. When artificial ants choose a path, it is based on the function (1).

If $X=\left\{X_{i} \mid X_{i}=\left(x_{i 1}, x_{i 2}, \ldots x_{i m}\right), i=1,2,3, \ldots, N\right\}$ is the data set of the clustering problem to be analyzed, $r$ is the clustering radius, $p h_{y e}(t)$ is the pheromone concentration on the path from data $X_{y}$ to data $X_{e}$ at time $t, d_{y e}$ represents the weighted Euclidean distance between the sample and the cluster center, and $p$ is a weighting factor, which can be determined according to the degree of image of each component in the cluster, then there is the following equation:

$$
d_{y e}=P\left(X_{y}-X_{e}\right)=\sqrt{\sum_{k=1}^{\mathrm{m}} \mathrm{P}_{k}\left(x_{y k}-x_{y k}\right)^{2}} .
$$

$p h_{y e}(0)=0$ is set as the initial information volume, then there is the following equation:

$$
p h_{y e}(0)= \begin{cases}1, & d_{y e} \leq r, \\ 0, & d_{y e}>r .\end{cases}
$$

According to the information concentration on the path between the sample and the cluster center, the probability $p h_{y e}$ that $X_{y}$ merges into $X_{e}$ is the following equation:

$$
p h_{y e}= \begin{cases}\frac{p h_{y e}^{a}(t) \eta_{y e}^{\beta}(t)}{\sum_{s \in S} p h_{y s}^{a}(t) \eta_{y s}^{\beta}(t)}, & j \in S, \\ O, & \text { other. }\end{cases}
$$

In equation (2), $\eta_{y e}(t)$ is a heuristic guiding function, which represents the expected degree of ant $X_{y}$ to $X_{e}$. The heuristic function is used to reflect the degree of similarity between pixels.
The larger the heuristic function, the greater the probability that the pixels belong to the same cluster. $\alpha$ and $\beta$ are the information accumulated in the process of pixel clustering and the image factors of the path selection by the heuristic guiding function, respectively. $S=\left\{X_{S} \mid d_{s e} \leq r, s=1,2, \ldots, j, j+1, \ldots, N\right\}$ is a set of feasible paths.

If $p h_{y e}(t) \geq p_{0}$, then $X_{y}$ is merged into the $X_{e}$ domain. It is set that $C_{j}=\left\{X_{k} \mid d_{k e} \leq r, k-1,2, \ldots, e\right\}$, and $C_{e}$ represents all the data sets merged into the $X_{e}$ domain. The ideal cluster center is found, which is expressed as follows:

$$
\overline{c e}=\frac{1}{E} \sum_{k=1}^{J} X_{k} \text {. }
$$

In equation (4), $X_{K} \in C_{e}$.

As the judgement ants move, the amount of pheromone on each path changes after a cycle, and the pheromone on each path is adjusted according to the global adjustment rule as follows:

$$
p h_{y e}(t)=\rho p h_{y e}(t)+\Delta p h_{y e} .
$$

In equation (5), $\rho$ is the attenuation coefficient of the pheromone over time, generally about 0.5 to 0.99 , and $\Delta p h_{y e}$ is the increment of the path pheromone in this cycle:

$$
\Delta p h_{y e}=\sum_{k=1}^{N} \Delta p h_{y e}^{k}
$$

In equation (6), $\Delta p h_{y e}^{k}$ represents the amount of pheromone left in the path of the $k$ th ant in this cycle.

The ACO is applied to MRI to establish an ACO-MRI model, and the algorithm steps are shown in Figure 1.

2.4. Observation Indicators. The diagnosis methods based on the KNN algorithm and SVM algorithm were compared with the ACO-MRI diagnosis method designed in this research for comparative observation. Accuracy, sensitivity, and specificity were used as diagnostic performance evaluation indicators for different inspection methods $[16,17]$ :

$$
\begin{aligned}
\text { accuracy } & =\frac{\mathrm{TP}+\mathrm{TN}}{\mathrm{TP}+\mathrm{TN}+\mathrm{FP}+\mathrm{FN}}, \\
\text { sensitivity } & =\frac{\mathrm{TP}}{\mathrm{TP}+\mathrm{FN}}, \\
\text { specificity } & =\frac{\mathrm{TN}}{\mathrm{FP}+\mathrm{TN}} .
\end{aligned}
$$

In equation (7), TP is expressed as the true positive of the test result, $\mathrm{FN}$ is false negative, $\mathrm{FP}$ is false positive, $\mathrm{TN}$ is true negative, and the confusion map shown in Figure 2 is obtained.

2.5. Statistical Methods. SPSS 19.0 was employed for data statistics and analysis. Mean \pm standard deviation $(\bar{x} \pm s)$ was how measurement data were expressed, and percentage (\%) was how count data were expressed. One-way analysis of variance was used for pairwise comparison. The difference was statistically considerable with $P<0.05$. 


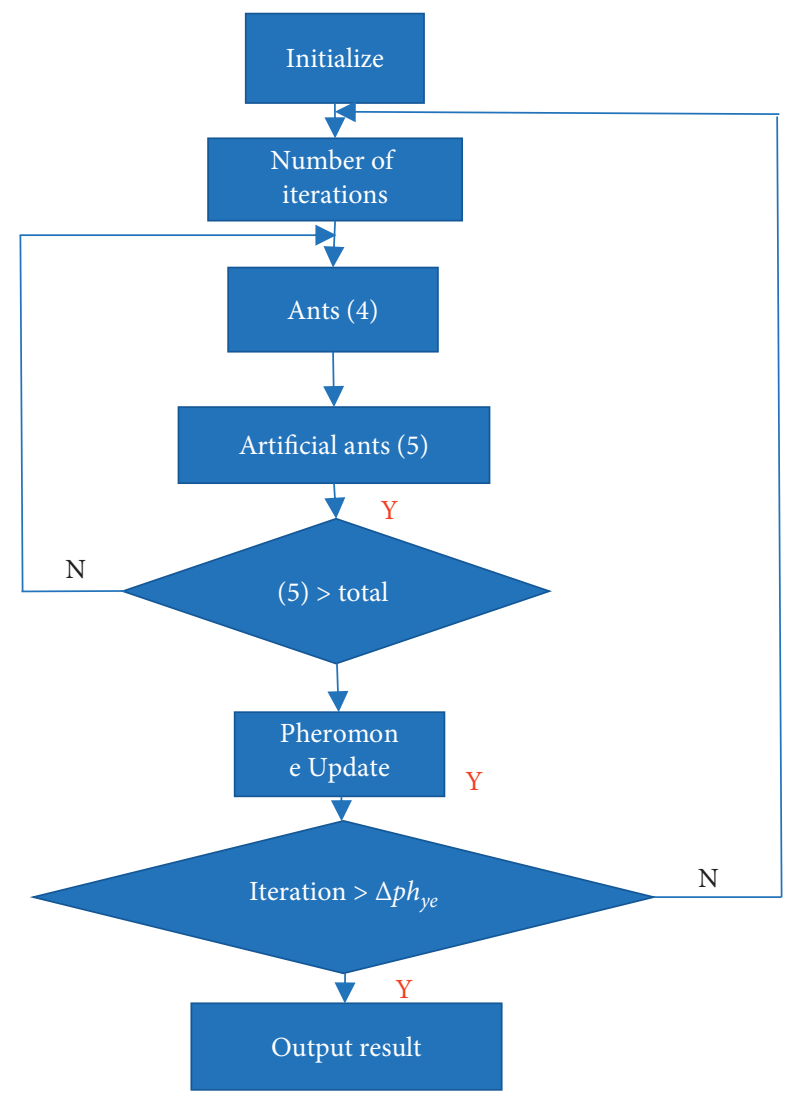

Figure 1: ACO algorithm steps.

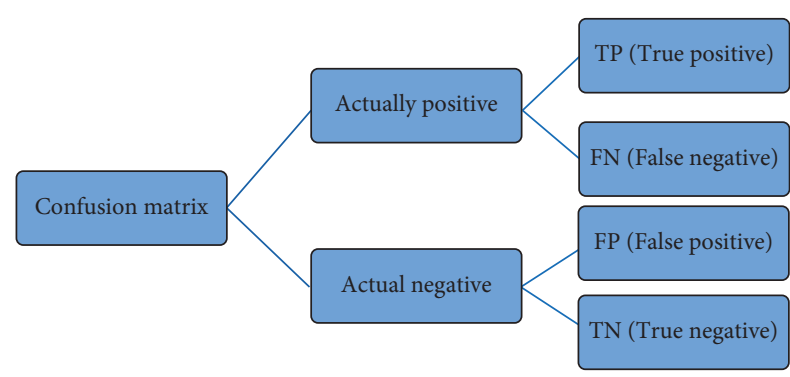

Figure 2: Confusion map.

\section{Results}

3.1. Pathological Comparison. A total of 175 patients were analyzed in this study, including 85 cases of invasive ductal carcinoma, 32 cases of invasive lobular carcinoma, 30 cases of mucinous carcinoma, and 42 cases of mixed carcinoma. The proportions are shown in Figure 3.

3.2. Algorithm Performance Analysis. The accuracy, sensitivity, and specificity of MRI imaging diagnosis of breast cancer based on the ACO algorithm were 93.6\%, 92.61\%, and $78.32 \%$, respectively. The accuracy, sensitivity, and specificity of the SVM algorithm in diagnosing breast cancer were $87.43 \%, 83.12 \%$, and $71.44 \%$, respectively. Those of the KNN algorithm in diagnosing breast cancer were $84.23 \%, 86.32 \%$,

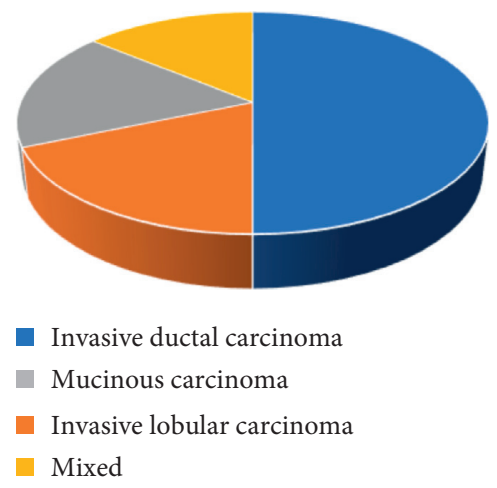

FIgURE 3: Comparison of pathological conditions of patients.

and $73.21 \%$, respectively. In Figure 4, the accuracy, sensitivity, and specificity of MRI based on the ACO algorithm in diagnosing breast cancer were higher than those of the KNN algorithm and the SVM algorithm. There was no statistically considerable difference in accuracy and sensitivity $(P>0.05)$, while the specificity of the ACO algorithm was greatly higher than that of the KNN and SVM algorithms $(P<0.05)$.

Figure 5 shows the comparison of the average gray path under the two numbers of ants. Figure 5(a) is the average gray path under the number of $1 / 5$ ants, and Figure $5(\mathrm{~b})$ is the gray path under the number of $1 / 8$ ants. The average gray path under the number of $1 / 5$ was greatly smaller than that under the number of $1 / 8$, and the difference between the two was substantial $(P<0.05)$.

Figure 6 shows the edge extraction of breast calcification points. The ACO was used to process massive breast images that contained calcification points and required to be judged by the doctor. After 30 iterations, the edge extraction of the breast calcification points of a female patient was obtained. The left side of Figure 6 is the edge pixels of the calcification points extracted from the digital image based on the ACO algorithm, and the right side is the original image. The leftright comparison showed that the ACO algorithm was clearer for the extracted images of the edges of breast cancer calcification points.

3.3. Comparison of MRI Imaging Features among Four Molecular Types of Breast Cancer. The comparison of breast MRI imaging features among the four molecular classification groups of breast cancer is shown in Figure 7. The chisquare test/Fisher exact probability method was used to analyze whether the overall distribution of breast MRI features in Luminal A, Luminal B, HER-2 overexpression, and TN was different. It turned out that there was no considerable difference in the overall distribution of tumor size, margin, and focus distribution among the four molecular typing groups $(P>0.05)$. However, there were considerable differences in the overall distribution of the three breast MRI imaging features of border, shape, and enhancement way among the four groups $(P<0.05)$.

3.4. Patient Imaging Data. Figure 8 shows the MR scan imaging data of a female patient, where Figure 8(a) is a TIWI 


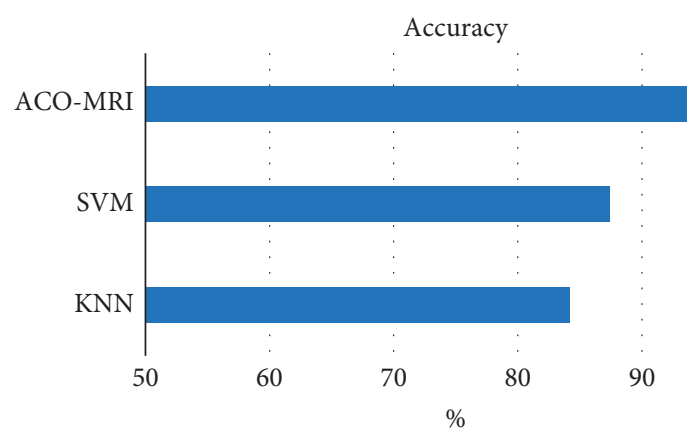

(a)

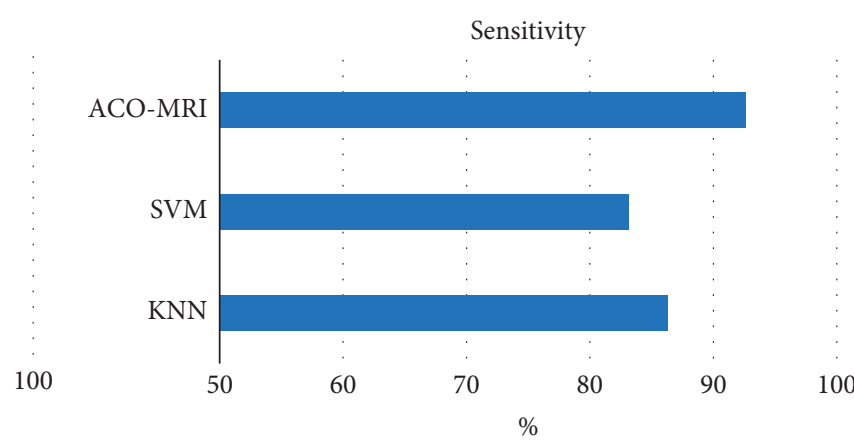

(b)

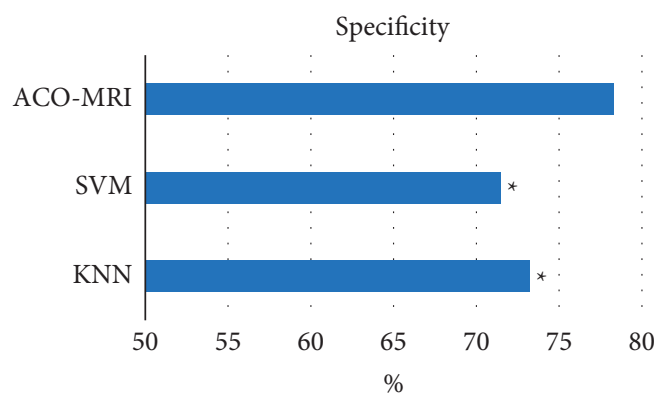

(c)

Figure 4: Comparison of accuracy, sensitivity, and specificity of three different diagnostic methods: (a) the accuracy of the three diagnostic methods; (b) the sensitivity of the three diagnostic methods; (c) the specificity of the three diagnostic methods. Note: ${ }^{*}$ indicates that the difference was considerable compared with ACO-MRI $(P<0.05)$.

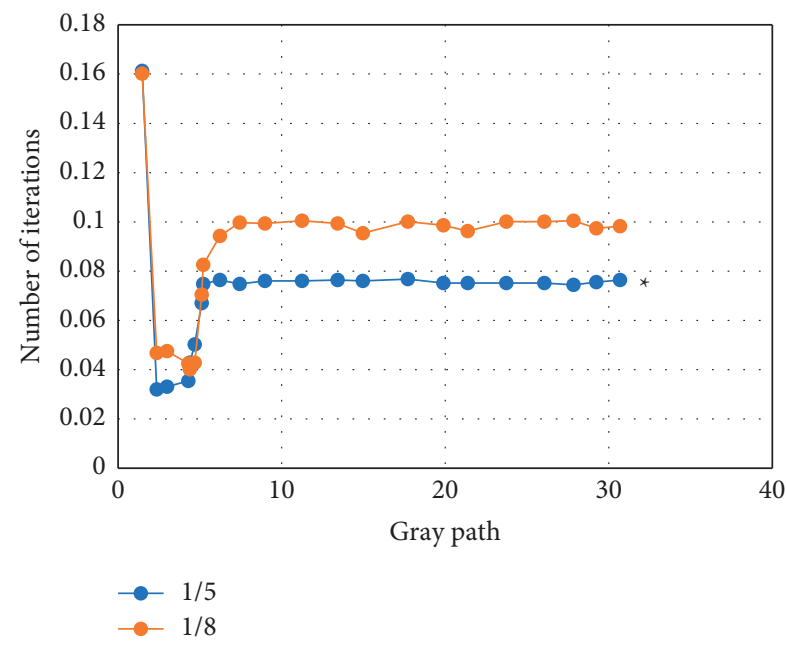

FIgURE 5: Comparison of the average gray path value under the number of two groups of ants. Note. ${ }^{*}$ means that the number of ants was considerable compared with $1 / 8$ ants $(P<0.05)$.

scan, Figure $8(\mathrm{~b})$ is a DWI scan, and Figure $8(\mathrm{c})$ is a T2WI scan. This patient had obvious calcification foci on the left breast, and the edge of the DWI image was relatively clear.

\section{Discussion}

So far, there are many studies on MRI in the diagnosis and treatment of breast cancer because MRI has a high resolution, and its imaging can clearly distinguish the lesions and

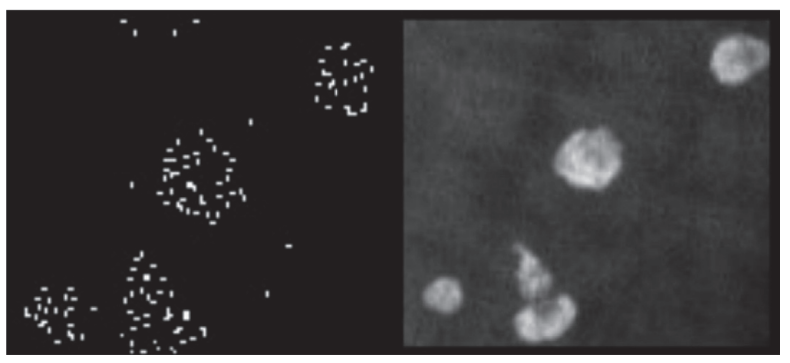

FIGURE 6: Edge extraction of breast calcification points in a female patient.

the surrounding mammary skin, glands, subcutaneous fat, and other tissues. This method is conducive to the detection of small lesions, puncture biopsy, and subsequent treatment $[18,19]$. As a new multidirectional and multisequence imaging technology, MRI is developing continuously and has great advantages in breast cancer detection, molecular typing, and prognosis assessment. However, this detection method still has some limitations and defects of missed diagnosis [20].

Wan et al. [21] explored the feature recognition, diagnosis, and prediction performance of semisupervised support vector machines for brain image fusion digital twins (DTs). For many unlabeled data in the brain image, the results showed that the model can provide $92.52 \%$ feature recognition and extraction accuracy. In view of the artificially caused inaccurate test results and missed diagnosis in breast cancer detection, an ACO-MRI diagnosis mode based on ACO in the 


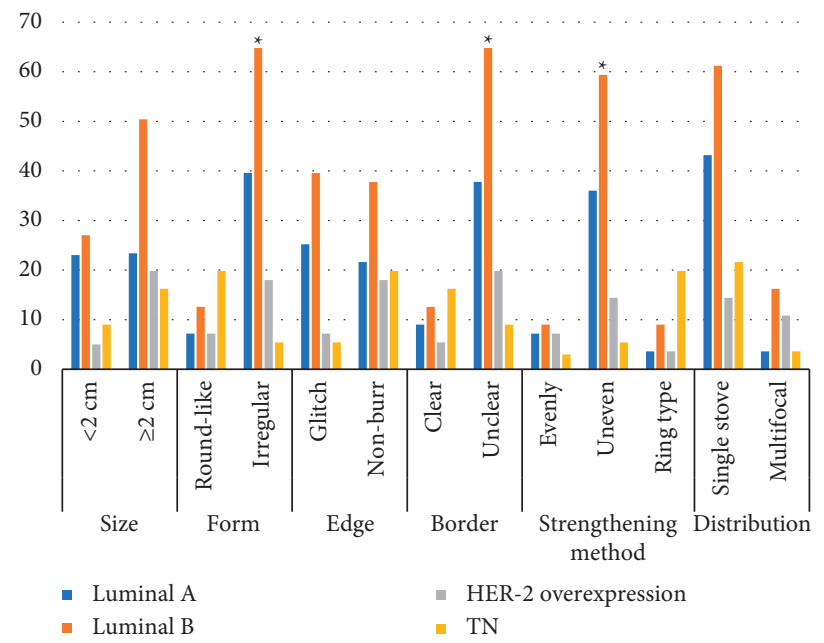

FIgURE 7: Comparison of MRI imaging features among the four molecular types of breast cancer. Note: ${ }^{*}$ indicates that the overall distribution difference between groups was considerable in terms of boundary, shape, and strengthening method $(P<0.05)$.

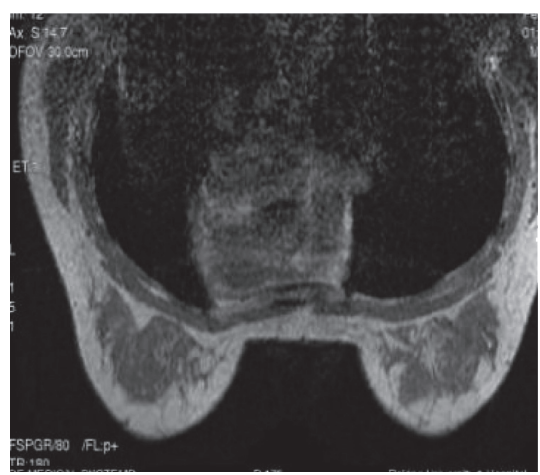

(a)

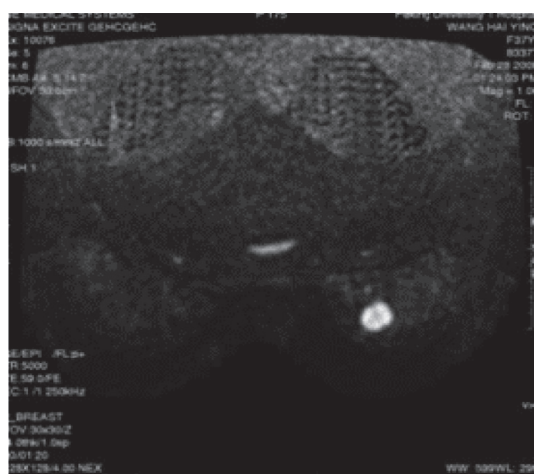

(b)

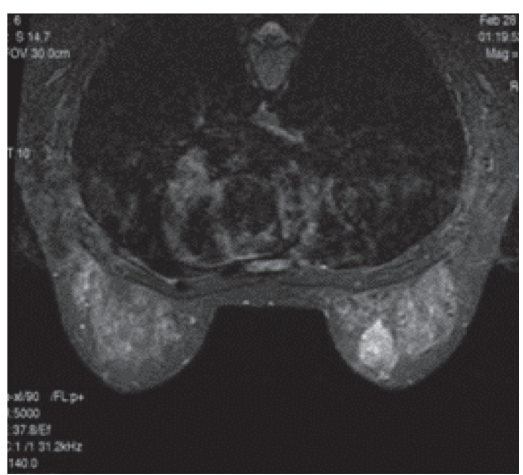

(c)

FIgURE 8: MR scan images of a female patient's breast: (a) T1WI scan; (b) DWI scan; (c) T2WI scan.

intelligent algorithm was proposed by combining ACOs from the field of intelligent algorithms with breast cancer datasets from real clinical trials, and the KNN algorithm and SVM algorithm were proposed and compared [22]. The accuracy, sensitivity, and specificity of the ACO algorithm in diagnosing breast cancer were $93.6 \%, 92.61 \%$, and $78.32 \%$, respectively, which were higher than those of the KNN algorithm and the SVM algorithm, and the specificity was statistically significant compared with the other two algorithms $(P<0.05)$. It was found that ACO algorithm mode had higher performance than the KNN and SVM algorithm [23, 24]. On the one hand, it was found that the grayscale path value was smaller with the more ants by comparing the average grayscale path value of the images under the two ant numbers. On the other hand, the edge of calcification points of breast lesions was extracted after 30 iterations of the ACO algorithm, and the image was clearer than the original image and had better recognition $[12,25,26]$.

Based on six MRI image characteristics including size, shape, edge, boundary, enhancement method, and lesion distribution, the overall distribution of MRI imaging features in Luminal A, Luminal B, HER-2 overexpression, and $\mathrm{TN}$ four breast cancer molecular types was compared. The results showed that the differences in the boundaries, morphology, and strengthening methods between the groups were statistically considerable, which was consistent with some of the previous results [27]. Yuan et al. [28] studied the correlation between the imaging characteristics of diffusion-weighted imaging (DWI) and dynamic contrast-enhanced magnetic resonance imaging (DCE-MRI) with molecular subtypes and prognostic factors of breast cancer. The results showed that the lobular sign, burr sign, strengthening method, time signal curve (TIC) type, and other parameters were related to prognostic factors and molecular subtypes. The lumps of luminal breast cancer mostly showed unclear borders and irregular shapes. The main reason was that luminal breast cancer was not highly malignant, and its histological grade was low. The tumor grew slowly and easily pulled with the fibers of the surrounding tissues, the boundaries were often unclear on imaging, and sometimes appeared as burrs, suggesting that the tumor had a lower aggressive behavior. 


\section{Conclusion}

In this research, a new method was proposed for breast detection, which used MRI combined with the ACO algorithm to extract breast calcification foci and shape recognition. The ANN algorithm and SVM algorithm for breast cancer diagnosis were compared. The experimental results showed that the ACO algorithm had better diagnostic performance than the ANN and SVM algorithm. In the clinical application of breast cancer detection and diagnosis, it can help clinicians to make better and effective decisions. However, when ACO is used to process images, the image has a large number of pixels and the number of ants, resulting in a large amount of computation. Moreover, the time will increase after several iterations. When the number of iterations is small, the extraction of edge points in the target image is not obvious enough, and there are some omissions of edge points. Therefore, this study has certain defects in terms of time consumption. Too large image will double the amount of computation, slow down the calculation speed, and even cause the device to be stuck. Therefore, this method can only be applied to images with small size, and further research is needed to improve the algorithm. In conclusion, this method is a good demonstration of the combination of the intelligent algorithm and MRI diagnosis of breast cancer. On the one hand, it improves the accuracy and efficiency of breast cancer detection, and on the other hand, it provides a new idea for realizing intelligent breast cancer detection. In the future, further optimization and improvement of the intelligent algorithm are required, which can make it have better application value in breast cancer diagnosis and early detection and treatment.

\section{Data Availability}

The data used to support the findings of this study are available from the corresponding author upon request.

\section{Conflicts of Interest}

The authors declare that there are no conflicts of interest.

\section{Authors' Contributions}

Shuang Liu and Min Tang contributed equally to this work.

\section{Acknowledgments}

This work was supported by Chongqing People's Hospital Project Fund (2016MSXM16).

\section{References}

[1] B. Khorram and M. Yazdi, "A new optimized thresholding method using ant colony algorithm for MR brain image segmentation," Journal of Digital Imaging, vol. 32, no. 1, pp. 162-174, 2019.

[2] A. Veloz, A. Weinstein, S. Pszczolkowski et al., "Ant colony clustering for ROI identification in functional magnetic resonance imaging," Computational Intelligence and Neuroscience, vol. 2019, Article ID 5259643, 2019.
[3] P. Stodola, K. Michenka, J. Nohel, and M. Rybanský, "Hybrid algorithm based on ant colony optimization and simulated annealing applied to the dynamic traveling salesman problem," Entropy, vol. 22, no. 8, p. 884, 2020.

[4] S. Yousefi, R. Azmi, and M. Zahedi, "Brain tissue segmentation in MR images based on a hybrid of MRF and social algorithms," Medical Image Analysis, vol. 16, no. 4, pp. 840-848, 2012.

[5] M. Taherdangkoo, M. H. Bagheri, M. Yazdi, and K. P. Andriole, "An effective method for segmentation of MR brain images using the ant colony optimization algorithm," Journal of Digital Imaging, vol. 26, no. 6, pp. 1116-1123, 2013.

[6] X. Dai, S. Long, Z. Zhang, and D. Gong, "Mobile robot path planning based on ant colony algorithm with $A^{*}$ heuristic method," Frontiers in Neurorobotics, vol. 13, p. 15, 2019.

[7] S. Partovi, D. Sin, Z. Lu et al., "Fast MRI breast cancer screening - ready for prime time," Clinical Imaging, vol. 60, no. 2, pp. 160-168, 2020.

[8] E. M. S. Negrão, J. A. Souza, E. F. Marques, and A. G. V. Bitencourt, "Breast cancer phenotype influences MRI response evaluation after neoadjuvant chemotherapy," European Journal of Radiology, vol. 120, Article ID 108701, 2019.

[9] K. Pinker, T. H. Helbich, and E. A. Morris, "The potential of multiparametric MRI of the breast," British Journal of Radiology, vol. 90, no. 1069, Article ID 20160715, 2017.

[10] C. K. Kuhl, “Abbreviated magnetic resonance imaging (MRI) for breast cancer screening: rationale, concept, and transfer to clinical practice," Annual Review of Medicine, vol. 70, no. 1, pp. 501-519, 2019.

[11] A. S. Tagliafico, M. Piana, D. Schenone, R. Lai, A. M. Massone, and N. Houssami, "Overview of radiomics in breast cancer diagnosis and prognostication," The Breast, vol. 49, pp. 74-80, 2020.

[12] L. F. Si, X. J. Liu, K. Y. Yang, L. Wang, and T. Jiang, “3.0 T MR diffusion tentor imaging in the differential diagnosis of breast mass lesions," Zhonghua Yixue Zazhi, vol. 96, no. 19, pp. 1510-1514, 2016.

[13] S. C. Partridge, N. Nissan, H. Rahbar, A. E. Kitsch, and E. E. Sigmund, "Diffusion-weighted breast MRI: clinical applications and emerging techniques," Journal of Magnetic Resonance Imaging, vol. 45, no. 2, pp. 337-355, 2017.

[14] R. M. Mann, R. Hooley, R. G. Barr, and L. Moy, "Novel approaches to screening for breast cancer," Radiology, vol. 297, no. 2, pp. 266-285, 2020.

[15] D. Sheth and M. L. Giger, "Artificial intelligence in the interpretation of breast cancer on MRI," Journal of Magnetic Resonance Imaging, vol. 51, no. 5, pp. 1310-1324, 2020.

[16] B. G. Choi, S.-W. Rha, S. W. Kim, J. H. Kang, J. Y. Park, and Y.-K. Noh, "Machine learning for the prediction of new-onset diabetes mellitus during 5-year follow-up in non-diabetic patients with cardiovascular risks," Yonsei Medical Journal, vol. 60, no. 2, pp. 191-199, 2019.

[17] H. Zhang, T. Fu, Z. Zhang, Z. Fan, C. Zheng, and B. Han, "[Application of support vector machine-recursive feature elimination algorithm in Raman spectroscopy for differential diagnosis of benign and malignant breast diseases]," Zhonghua Zhongliu Zazhi, vol. 36, no. 8, pp. 582-586, 2014.

[18] S. H. Sun, C. W. Zhou, L. Y. Zhao, R. Z. Zhang, and H. Ouyang, "[Texture analysis based on contrast-enhanced MRI can predict treatment response to neoadjuvant chemotherapy of breast cancer]," Zhonghua Zhongliu Zazhi, vol. 39, no. 5, pp. 344-349, 2017.

[19] X. Wang, W. Wang, J. Wang, Y. Song, X. Wang, and F. Ye, "[Clinical application of MRI-guided puncture of breast 
microlesions]," Zhonghua Zhongliu Zazhi, vol. 37, no. 9, pp. 682-685, 2015.

[20] X. Yang, G. Liu, X. Yu, Q. Zhang, W. Zhang, and Y. Guo, "[Controlled study of gadobenate dimeglumine versus gadopentetate in breast tumor MR dynamic enhancement scanning]," Zhonghua Yixue Zazhi, vol. 95, no. 25, pp. 2003-2005, 2015.

[21] Z. Wan, Y. Dong, Z. Yu, H. Lv, and Z. Lv, "Semi-supervised support vector machine for digital twins based brain image fusion," Frontiers in Neuroscience, vol. 15, Article ID 705323, 2021.

[22] E. Y. Chae, H. J. Shin, H. J. Kim et al., "Diagnostic performance of automated breast ultrasound as a replacement for a hand-held second-look ultrasound for breast lesions detected initially on magnetic resonance imaging," Ultrasound in Medicine and Biology, vol. 39, no. 12, pp. 2246-2254, 2013.

[23] A. Boss, L. Rohrer, and N. Berger, "Neue wege in der Senologischen bildgebung," Therapeutische Umschau, vol. 77, no. 2, pp. 81-84, 2020.

[24] Y. J. He, X. T. Li, Z. Q. Fan et al., "[Application of decision curve on evaluation of MRI predictive model for early assessing pathological complete response to neoadjuvant therapy in breast cancer]," Zhonghua Yixue Zazhi, vol. 98, no. 4, pp. 260-263, 2018.

[25] T. T. Sun, W. H. Liu, Y. Q. Zhang, L. H. Li, R. Wang, and Y. Y. Ye, "[Diagnostic value of quantitative pharmacokinetic parameters and relative quantitative pharmacokinetic parameters in breast lesions with dynamic contrast-enhanced MRI]," Zhonghua Yixue Zazhi, vol. 97, no. 29, pp. 2266-2270, 2017.

[26] L. Xin, Q. Liu, L. Xu et al., "[Role of magnetic resonance imaging for response evaluation and predictive value of tumor biomarkers in the neoadjuvant chemotherapy for breast cancer: a multi-center prospective study]," Zhonghua Yixue Zazhi, vol. 94, no. 26, pp. 2018-2021, 2014.

[27] Y. Ji, H. Li, A. V. Edwards et al., "Independent validation of machine learning in diagnosing breast Cancer on magnetic resonance imaging within a single institution," Cancer Imaging, vol. 19, no. 1, p. 64, 2019.

[28] C. Yuan, F. Jin, X. Guo, S. Zhao, W. Li, and H. Guo, "Correlation analysis of breast cancer DWI combined with DCEMRI imaging features with molecular subtypes and prognostic factors," Journal of Medical Systems, vol. 43, no. 4, p. 83, 2019. 